\title{
La educación para la ciudadanía global como certeza para un futuro complejo e incierto
}

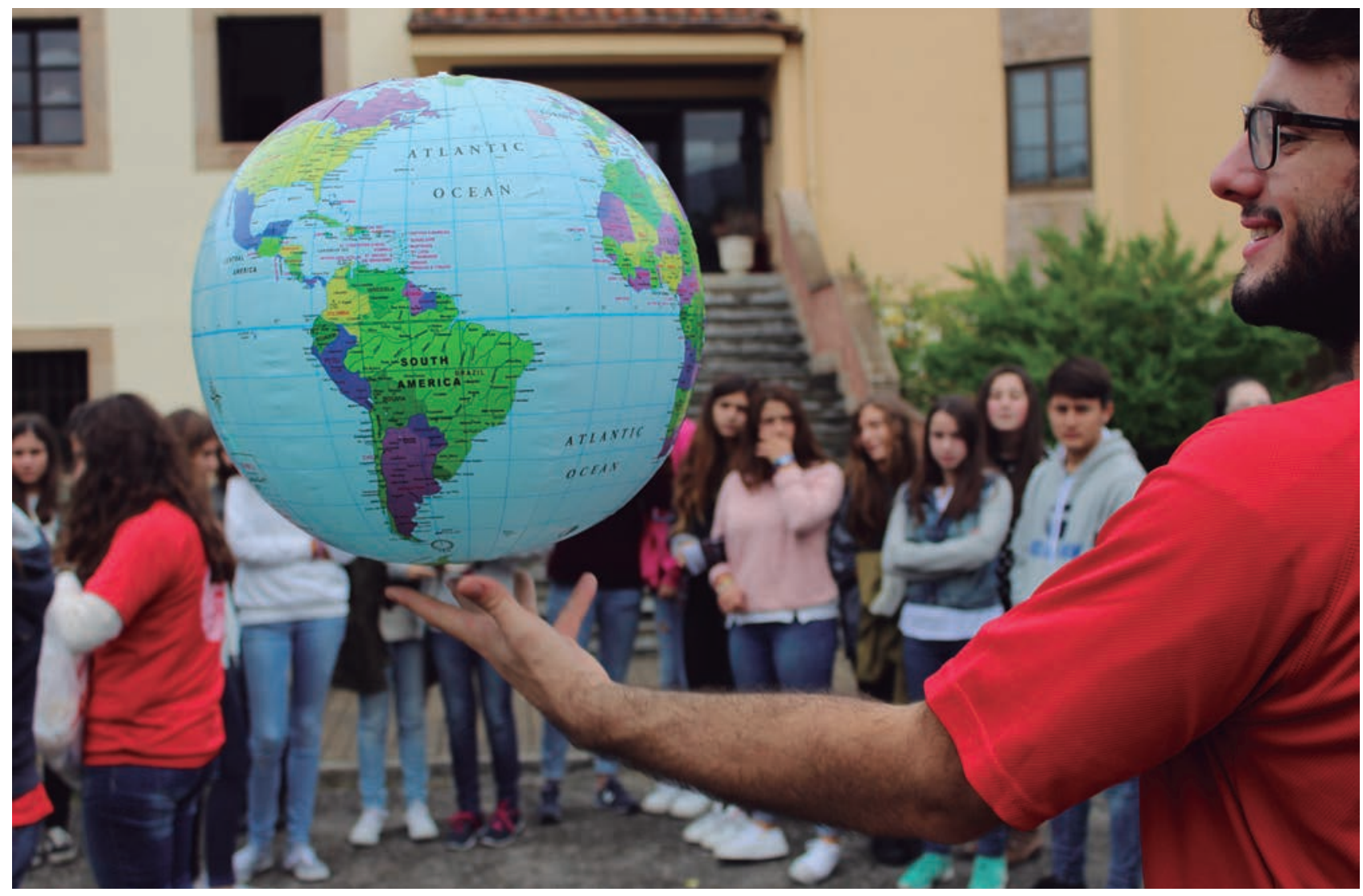

Esta crisis pone en evidencia las carencias de una educación ajena a la realidad global en la que vivimos. Proponemos la promoción de una ciudadanía activa y comprometida, que sea capaz de vivir su vida de forma interconectada en escenarios locales y globales más justos y solidarios. La educación para la ciudadanía global es nuestra propuesta para responder tanto a la crisis actual como a los retos globales que van a

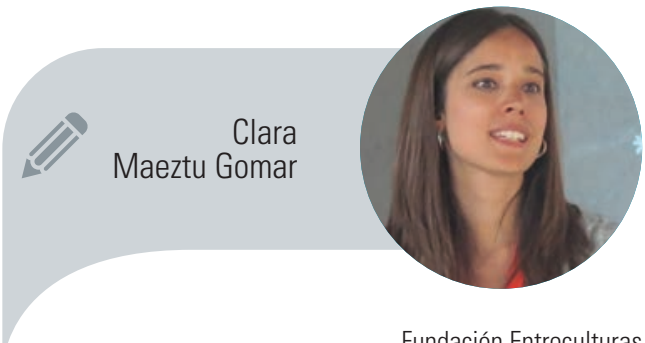

Fundación Entreculturas c.maeztu@entreculturas.org

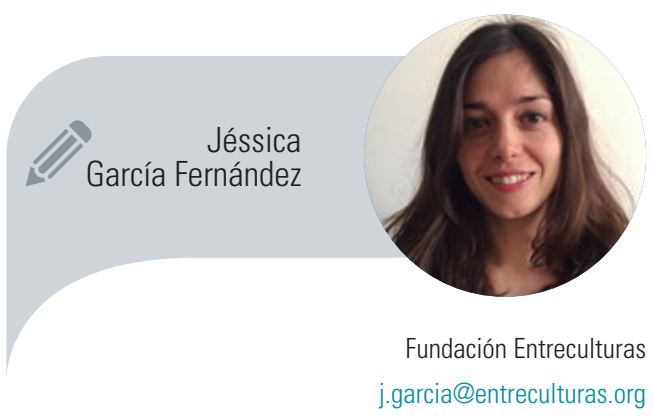
interpelarnos en el futuro. 


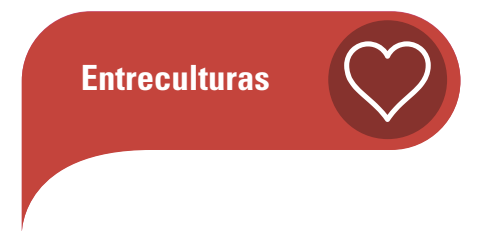

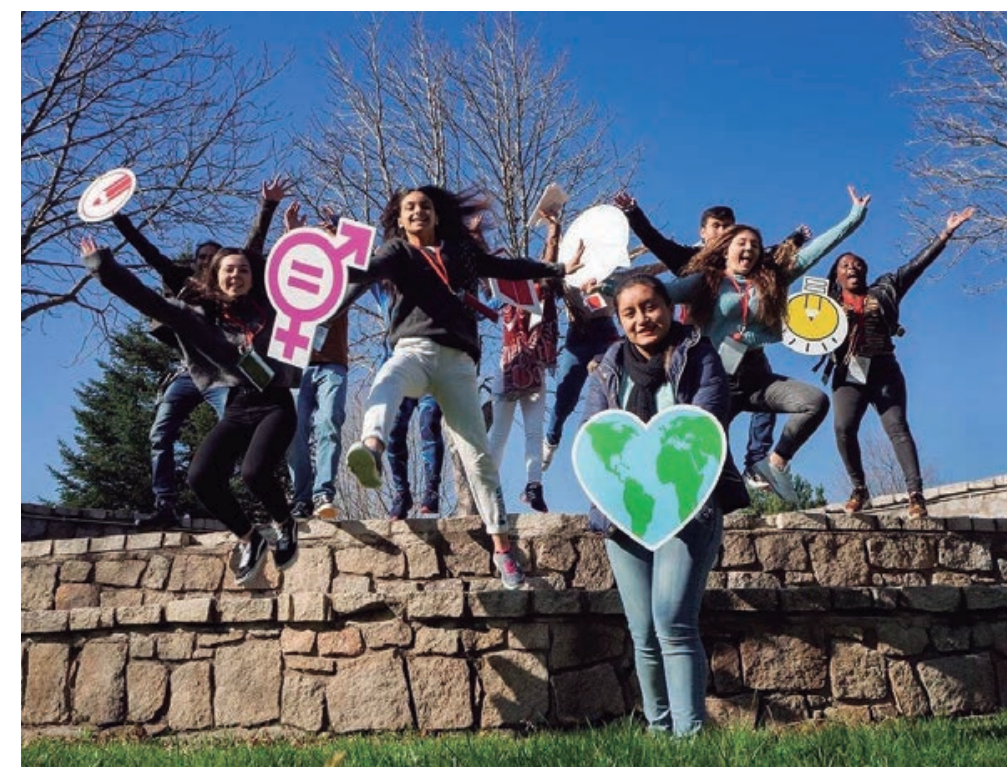

Jóvenes de más de 13 países participantes en el VII Encuentro Global de la Red Solidaria de Jóvenes de Entreculturas
Estamos conectados. Somos interdependientes. Es ya mucho más lo que nos une que lo que nos separa. Estos son algunos de los principales aprendizajes que nos está dejando esta COVID-19. Los retos globales afectan al mundo en su conjunto. Las problemáticas son globales y las soluciones, por tanto, también tienen que serlo. Es cierto que los efectos no serán los mismos en todos los lugares, pero lo que sí sabemos es que todas y todos nos veremos afectadas. Y entonces, si todo se conecta, ¿pueden estar las personas desconectadas? ¿Podemos ocuparnos únicamente de asuntos individuales, olvidando las redes de solidaridad, de cuidados y de gestión de lo común? ¿Son las redes económicas, de producción, extracción y explotación las únicas posibles? ¿La educación puede desconectarse de la realidad global en la que se inserta? ¿Qué papel juega en este nuevo escenario global?

Quizás algunas de estas preguntas encuentren sus respuestas en procesos ya iniciados y que desde las organizaciones de desarrollo venimos defendiendo desde hace tiempo: la educación para la ciudadanía global.

Una educación para la ciudadanía global que ha pasado en estos últimos años, gracias a las organizaciones y al apoyo de diferentes agentes públicos y privados, a ser una competencia evaluada en los informes PISA. Progresivamente, las sociedades civiles y los Gobiernos han ido tomando conciencia de aquello que los movimientos, colectivos y organizaciones sociales planteamos hace décadas y que hoy se hace más patente que nunca: la interdependencia global es el signo distintivo de nuestra época. Y esto conlleva numerosos retos que afectan directamente a la educación en su función socializadora y en su capacidad de dar respuesta a los retos que hoy nos plantea la COVID-19.

\section{¿De qué hablamos cuando hablamos de educación para la ciudadanía global?}

Desde Entreculturas, entendemos la ciudadanía como un derecho fundamental que tenemos todas las personas como iguales, corresponsables en la sostenibilidad y bienestar de la comunidad global a la que pertenecemos, teniendo en cuenta especialmente la inclusión y la participación de colectivos y personas más vulnerables. Desde nuestra propuesta, buscamos promover una ciudadanía local y global, intercultural, conectada con la Tierra, promotora de convivencia pacífica, igualitaria y articulada en movimientos de transformación desde las comunidades. Para construir esta ciudadanía global, apostamos por la educación como derecho y estrategia clave, de la mano de la movilización social y la incidencia política para la transformación social. Esto nos lleva a la promoción de la educación para la ciudadanía global como enfoque y metodología en procesos de participación orientados al crecimiento personal integral, para favorecer la inclusión de todas las personas e impulsar su compromiso como agentes de cambio en su propia vida y en el mundo. Una educación que promueva las experiencias necesarias desde la infancia, para que las personas podamos adquirir los conocimientos, herramientas y actitudes necesarios para actuar como agentes de cambio en nuestros entornos, articulados en redes para el trabajo por la transformación social y la configuración de un mundo justo e igualitario. Una educación, por tanto, ubicada y contex- 
tualizada en la realidad global en la que se inserta, que ofrezca una experiencia integral de crecimiento y desarrollo.

«El objetivo de la actividad en la que participamos era reivindicar la importancia de dar voz y plena participación a los y las jóvenes; quienes, mediante talleres y momentos de reflexión individual y común, debíamos tratar algunas de las problemáticas más importantes que afronta la sociedad abordando los desafíos mundiales a los que se enfrenta el planeta, a 10 años de alcanzar la Agenda por la Igualdad y el Desarrollo Sostenible 2030. Y eso justo es lo que destacamos nosotros, los jóvenes. El habernos ayudado mutuamente a salir de esta burbuja en la que vivimos, el despertar con sabor agridulce, pero sabiendo que no estamos solos. Nos encontramos en los otros, ahora somos un aviso a tiempo, un abrazo de bienvenida».

LAURA Bonet, participante valenciana en el proyecto Red Solidaria de Jóvenes

Además, desde esta propuesta queremos contribuir a que la educación para la ciudadanía global se incluya en la educación formal de manera integral, tanto a través de la regulación educativa vigente como de la práctica socioeducativa en centros escolares y entidades. También promovemos la inclusión de la misma en las experiencias de educación no formal y en la articulación de las comunidades - cuidando especialmente la participación de las personas y colectivos más excluidos-, con mirada local y global y desde la interculturalidad, entendida como oportunidad de aprendizaje y parte de procesos de crecimiento comunitario. Queremos así favorecer la cohesión social, la inclusión y el fortalecimiento de una ciudadanía orientada al bienestar de todas las personas que en estos momentos y tras la vuelta a los centros educativos será tan necesaria.

«En el transcurso de las presentaciones de los líderes Wiwa de la región de Sierra Nevada en Colombia, escuchando cada uno de los relatos, mi sentimiento fue de indignación y surgió con más fuerza ese deseo de la transformación social y el pensar en el otro. A partir de ahí trabajamos apuestas para la construcción de la ciudadanía global, desde la defensa del territorio,

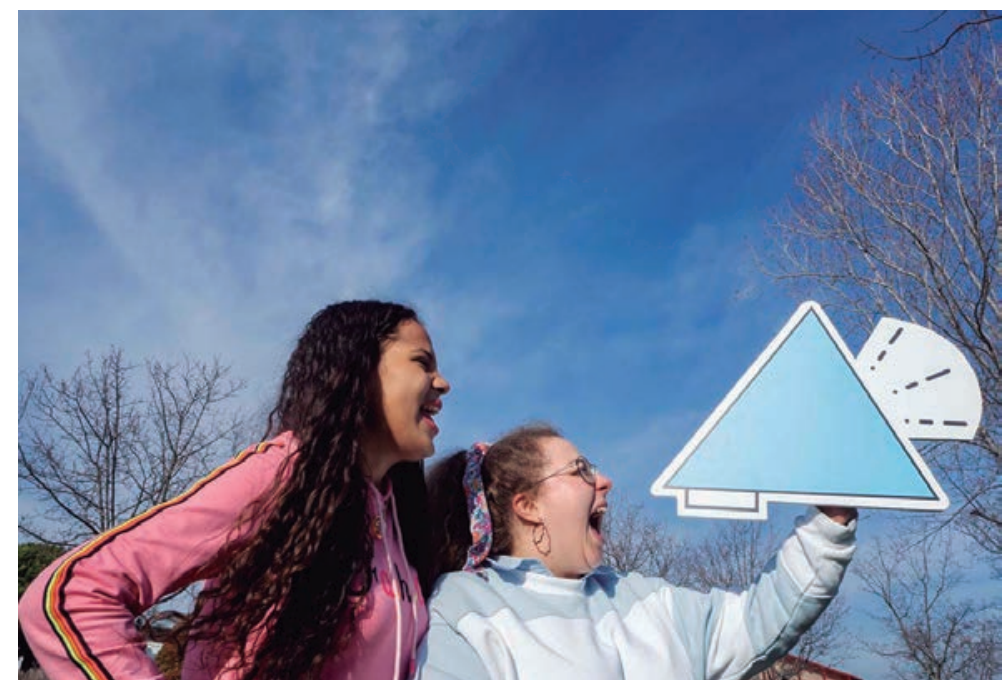

ya que es en este donde se fortalecen las dinámicas sociales y donde se crean los lazos comunitarios que son muy importantes para este proceso".

Víctor de Jesús Padilla, líder de proyectos comunitarios y joven participante de la Red Generación 21+ en Fe y

Alegría Colombia

\section{¿Cómo contribuye la ciudadanía global para hacer frente a la postcrisis?}

Desde Entreculturas proponemos una fase de reconstrucción que apueste por:

入 Una educación que facilite experiencias para fortalecer una ciudadanía informada, crítica, comprometida y activa sobre los retos globales de nuestro tiempo, que en momentos como el actual, actúe como un solo cuerpo sin cerrar los ojos a lo que ocurre en ninguna de sus partes, con especial foco en las personas más excluidas.

خ Una ciudadanía capaz de articularse con otras personas y colectivos en todo el mundo a través de la pedagogía del encuentro y la creación de redes, que incida en lo local para transformar lo global. Promoviendo en ambos ámbitos las relaciones justas y equitativas entre las personas y con el planeta, para así garantizar la supervivencia de ambos.

$\boldsymbol{\lambda}$ Una educación que facilite herramientas, habilidades y recursos concretos para actuar sobre la realidad, para reconstruirnos emocionalmente y conectar nuestras emociones y acciones con aquello que ocurre a nuestro alrededor y sobre lo que cada quien tiene la responsabilidad de actuar.
Jóvenes de la Red Generación 21+ participando en acciones de incidencia en el VII Encuentro Global de la Red Solidaria de Jóvenes de Entreculturas 


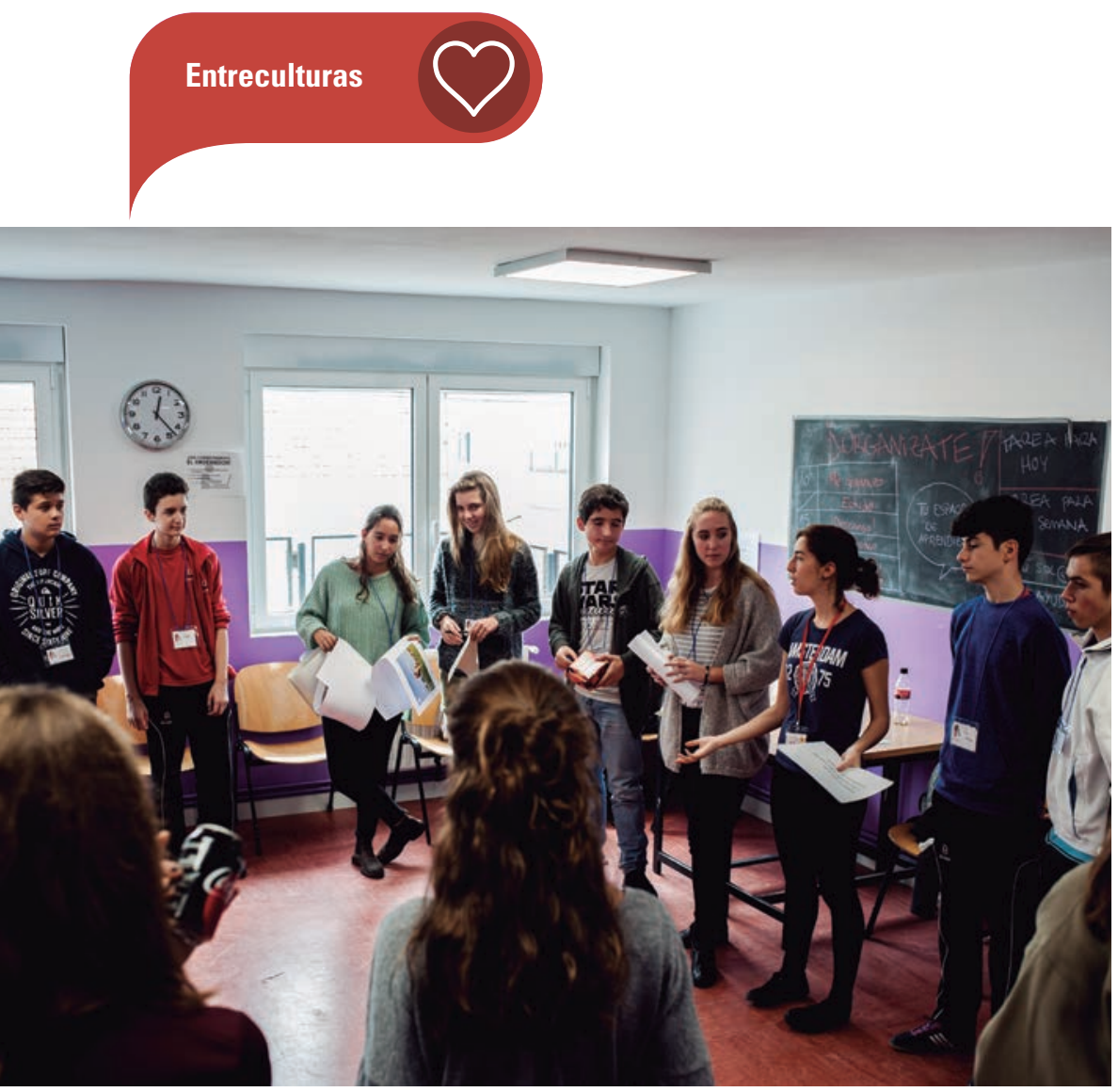

Jóvenes de la Red Generación 21+ participando en acciones de incidencia en el VII Encuentro Global de la Red Solidaria de Jóvenes de Entreculturas
"Tras esta experiencia nos sentimos personas diferentes, más motivadas para crear una nueva identidad para el mundo, ique precisa de cambios urgentes! Aprendimos muchísimo y tenemos como objetivo aprender más y más, iy queremos mostrar a las personas de nuestro país que hay mucho más por ver y hacer! Sentimos una sensación de esperanza, algo que no sentíamos desde hacía mucho tiempo: jesperanza! Vemos que no estamos solos en esta lucha, y que podemos empezar el cambio. [...] Nos gustaría dejar un mensaje al Mundo: intentaremos hacer de ti un lugar mejor".

Pedro Machado y Diogo Maroues, alumnos de la Escola Secundária do Lumiar en Portugal, tras su participación en el VII Encuentro Global de la Red Solidaria de Jóvenes

\section{La educación para la ciudadanía global en el marco político}

La apuesta por la educación para la ciudadanía global no ha pasado desapercibida en la Agenda Global de Desarrollo Sostenible, que configura el objetivo de desarrollo sostenible 4, relacionado con la educación, como un objetivo clave y palanca para posibilitar el desarrollo de toda la agenda. Además, se posiciona en qué tipo de educación debe abordarse en su meta 4.7, nombrando específicamente el enfoque de educación para la ciudadanía global. Tampoco a la coordinadora estatal de ONG, que en su documento 20 medidas para hacer frente a la pandemia del COVID-19 desde el sistema de cooperación para el desarrollo y las ONGD defiende lo siguiente: "en materia de Educación para la Ciudadanía Global (EpCG), es necesario un compromiso de la Agencia Estatal de Cooperación al Desarrollo para apoyar la continuidad y refuerzo del trabajo de Educación para el Desarrollo y la Ciudadanía Global durante y de manera posterior a la emergencia para multiplicar y extender la promoción de los valores de la solidaridad, cooperación y el internacionalismo entre la sociedad española. Estas son herramientas clave en la lucha contra la pandemia y por la justicia global".

"La educación no es un privilegio. Debemos buscar recursos y herramientas para que llegue a todos y todas. La crisis de la COVID-19 no solo afecta económicamente. Pensemos en todos los estratos de la sociedad".

Reflexión de una joven para la campaña "La mejor lección" de la Campaña Mundial por la Educación

«Me quedo con la oportunidad y la confianza en nosotros. Cuando crees en una causa todo resultará fácil para ti. Si crees en un cambio harás todo lo que esté en tu mano para cambiarlo. Cualquiera es capaz de cambiar esta realidad, simplemente hace falta la conciencia y el cuestionamiento. Tomar un papel de responsabilidad y acción».

Alicia del ReAL, participante en el proyecto de aprendizaje servicio "Miradas que Migran"

\section{Conclusiones: un enfoque educativo inaplazable}

La crisis de la COVID-19 pone en evidencia, una vez más, que va a ser difícil seguir sosteniendo una educación ajena, en los contenidos tratados y las habilidades fomentadas, a la realidad global en la que vivimos. El escenario global que va a quedar a consecuencia de esta crisis es complejo, y una respuesta responsable al mismo va a requerir el fomento de conocimientos y habilidades para la promoción de una ciudadanía activa y comprometida, que sea capaz de vivir su vida de forma interconectada con otros y otras, en la tarea de contribuir a configurar escenarios locales y globales más justos y solidarios. Si no asumimos la centralidad de la educación en estos momentos y apostamos por un enfoque educativo que sea 


\section{ACTIVIDADES DE AULA}

capaz de atender a esta necesidad, estaremos dejando sin herramientas a las futuras generaciones, formadas solo en el ámbito académico y para el ejercicio de profesiones, en un futuro global cada vez más incierto y complejo que necesita una ciudadanía informada, activa y comprometida con la construcción de un mundo más justo.

En este sentido, consideramos que el enfoque de educación para la ciudadanía global lleva años alumbrando el camino para incluir los retos globales relacionados con los derechos humanos, la defensa del medioambiente y de lo común, la igualdad de género o la interculturalidad en la práctica educativa formal y no formal, al tiempo que promueve experiencias de participación social para articular a las comunidades a nivel internacional. Puede que la situación actual, y los retos del nuevo escenario global que se están perfilando, sean una oportunidad única para trabajar por este modelo de educación transformadora. La defensa de este modelo de educación, para quienes aún creemos que la educación es la llave del desarrollo y la transformación social, debe ser en este tiempo más decidida que nunca.

«Me he dado cuenta de que la mayoría de la gente se ha volcado a ayudar, se ha creado un poco ese sentimiento de unidad. De todo se puede aprender y lograr tener una educación transformadora donde las jóvenes y los jóvenes puedan aprender a educar su corazón y su mente. En todos estos meses hemos puesto a prueba la resistencia del sistema educativo y de los derechos humanos, entre otros muchos. COVID-19, solo

\section{HEMOS HABLADO DE}

\section{Educación para la ciudadanía global; educación transformadora; jóvenes agentes de cambio; crisis globales; transformación social; COVID-19.}

Este artículo fue solicitado por PADRES Y MAESTROS en marzo de 2020, revisado y aceptado en mayo de 2020.
Situaciones como la generada por la COVID-19 y otras que puedan surgir en el futuro constituyen una oportunidad educativa privilegiada para trabajar con grupos sobre los retos globales que afectan al mundo en el que vivimos.

En Entreculturas creemos que se pueden poner en marcha un sinfín de actividades y procesos desde innumerables espacios de la educación formal y no formal. Para esto, sugerimos incorporar en su diseño las siguientes "cuatro íes":

У Investigar: recopilar información de forma crítica y contrastada sobre la situación que vivimos y sus consecuencias con enfoque global, atendiendo especialmente a cómo se está viviendo y afrontando en distintos lugares del mundo y las similitudes y diferencias que existen con el contexto propio.

У Interrelacionar: analizar esta información, identificando qué retos globales están involucrados en la misma y cómo se interrelacionan entre sí (deterioro del medioambiente, igualdad de género, interculturalidad, cultura de paz, derechos humanos, etc.)

У Iniciar: analizar qué iniciativas se están poniendo en marcha, tanto en otros países como en nuestros contextos más cercanos. ¿Qué ideas de acciones que se están poniendo en marcha en otras zonas puedo incorporar a mi contexto? ¿Con qué acciones de las ya existentes en mi entorno puedo empezar a contribuir? ¿Qué acciones que aún no se están realizando puedo contribuir a iniciar?

У Incorporar: analizar los aprendizajes que nos llevamos de esta situación, extrayendo qué podemos aprender de lo vivido, qué podremos aplicar a nuestro trabajo de transformación social una vez que el escenario global vuelva a cambiar. ¿A qué retos quiero dedicarme, y con qué nuevas herramientas, acciones y redes que he construido durante este tiempo? ¿Qué papel quiero tener de ahora en adelante como parte de la ciudadanía global en la que me inserto?

«La tormenta de una nueva pandemia nos tomó desprevenidos a todos; sin distinguir entre género, religión, nacionalidad o cualquier otro tipo de criterio discriminatorio, se fue expandiendo a lo largo de nuestra casa esférica y, sin querer, nos enseña mucho... ahora es nuestro deber aprender de ellorl.

Facunoo Velásouez, joven participante de la Red Generación 21+ desde el grupo de Protagonismo Juvenil Organizado de Salta (Argentina)

quiero decirte que he aprendido realmente el valor de

las cosas necesarias del amor verdadero y que una videollamada te puede llevar en un segundo a la otra punta del mundo. Estoy segura de que saldremos de esta juntas y juntos».

IRIS TRIGo, voluntaria de Entreculturas en Galicia •

\section{DARr SABEr más}

COORDINADORA ESTATAL DE ONG. (2019). 20 medidas para hacer frente a la pandemia de/ COVID-19. Recuperado de https://coordinadoraongd.org/2020/04/20-medidas-para-hacer-frente-a-la-pandemia-del-covid-19

FUNDACIÓn ENTRECULTURAS. (2019). Un mundo en tus manos: propuesta pedagógica para una ciudadanía global. Recuperado de https:// mundoentusmanos.org/

NACIONES Unidas. (2015). Agenda de Objetivos de Desarrollo Sostenible. Objetivo 4: garantizar una educación inclusiva, equitativa y de calidad. Recuperado de https://www.un.org/sustainabledevelopment/es/education/ 